\title{
与 $\mathcal{I}$ - 超滤相关的基数不变量
}

\author{
洪剑勇, 张树果* \\ 四川大学数学学院, 成都 610064 \\ E-mail: hjy1982715@yahoo.com.cn, shuguo1@yahoo.com.cn \\ 收稿日期: 2011-09-01; 接受日期: 2012-08-20; * 通信作者 \\ 国家自然科学基金 (批准号: 10971149) 资助项目
}

摘要 我们对自然数 $\omega$ 上的每一个理想 $\mathcal{I}$ 引入了一个新的基数不变量 $\operatorname{non}^{* *}(\mathcal{I})$. 我们证明相应的 $\mathcal{I}$ - 超滤的兼纳存在性可以用 $\operatorname{non}^{* *}(\mathcal{I})$ 与连续统 $\mathfrak{c}$ 的等式来刻画. 具体地, 我们有如下的结果:

（1）如果 $\operatorname{non}^{* *}(\mathcal{I})=\mathfrak{c}$, 那么任何一个由小于 $\mathfrak{c}$ 个集合生成的滤子都包含在某个 $\mathcal{I}$ - 超滤中.

(2) 存在一个滤子刚好可以由 non** $(\mathcal{I})$ 个集合生成, 但不包含在任何一个 $\mathcal{I}$ - 超滤中.

(3) 任何一个由小于 non** $(\mathcal{I})$ 个集合生成的超滤一定是 $\mathcal{I}$ - 超滤.

以上的结果是相应的 P- 点和 Ramsey 超滤的经典结论的一个推广. 我们将对一些具体的理想, 确 定 $\operatorname{non}^{* *}(\mathcal{I})$ 的大小, 具体地, 我们得到了 $\operatorname{non}^{* *}(\operatorname{Fin} \times$ Fin $)=\mathfrak{d}, \operatorname{non}^{* *}(\mathcal{E D})=\operatorname{cov}(\mathcal{M})$ 以及对不满足 Fin-BW 性质的理想 $\mathcal{I}$, 都有 $\operatorname{non}^{* *}(\mathcal{I}) \geqslant \mathfrak{d}$.

关键词 理想 $\mathcal{I}$ - 超滤 基数不变量

MSC (2010) 主题分类 03E17, 03E05

\section{1 引言}

$\mathcal{I}$ - 超滤首先是由 Baumgartner 最先引入的. 设 $\mathcal{I}$ 是集合 $X$ 上的一个理想, 自然数上的一个超滤 $\mathcal{U}$ 称为是 $\mathcal{I}$ - 超滤, 如果对任一个函数 $F: \omega \rightarrow X$, 都存在一个 $A \in \mathcal{U}$ 使得 $F[A] \in \mathcal{I}$. Baumgartner ${ }^{[1]}$ 和 Brendle ${ }^{[2]}$ 研究了 $X$ 是 Cantor 空间 $2^{\omega}$ 及 $\omega_{1}$ 情况下相应的一些拓扑版本的 $\mathcal{I}$ - 超滤和序数超滤 之间的关系. Flašková ${ }^{[3]}$ 研究了 $X$ 是自然数集 $\omega$ 的情况下的一些相应的 $\mathcal{I}$ - 超滤及经典的 P- 点和 Ramsey 超滤之间的关系. Flašková ${ }^{[4]}$ 把 P- 点和 Ramsey 超滤都刻画成 $\omega \times \omega$ 上的一些理想的 $\mathcal{I}$ - 超 滤, 具体的来说, Fin $\times$ Fin- 超滤就是 P- 点, 而 $\mathcal{E D}$ - 超滤就是 Ramsey 超滤.

$\mathrm{P}-$ 点和 Ramsey 超滤的存在性与基数不变量 $\mathfrak{d}$ 和 $\operatorname{cov}(\mathcal{M})$ 密切相关, 事实上, 我们有如下的经典 结果, 其中的 $\mathfrak{c}$ 表示连续统的基数.

定理 $1.1(1)^{[5]}$ a) 如果 $\mathfrak{c}=\mathfrak{d}$, 那么任何一个由小于 $\mathfrak{c}$ 个集合生成的滤子都包含在某个 P- 点中.

b) 存在一个滤子刚好可以由 $\mathfrak{d}$ 个集合生成, 但不包含在任何一个 P- 点中.

c) 任何一个由小于 $\mathfrak{d}$ 个集合生成的超滤一定是 P- 点.

(2) ${ }^{[6]}$ a) 如果 $\mathfrak{c}=\operatorname{cov}(\mathcal{M})$, 那么任何一个由小于 $\mathfrak{c}$ 个集合生成的滤子都包含在某个 Ramsey 超 滤中.

b) 存在一个滤子刚好可以由 $\operatorname{cov}(\mathcal{M})$ 个集合生成, 但不包含在任何一个 Ramsey 超滤中. 
证明可见文献 [7]. 那么与 $\mathcal{I}$ - 超滤相关的基数不变量是什么呢? 我们将在下一节中引入一个基数 不变量 $\operatorname{non}^{* *}(\mathcal{I})$ 并证明其相应的结论.

在第 3 节中, 我们将对一些具体的理想 $\mathcal{I}$, 确定 $\operatorname{non}^{* *}(\mathcal{I})$ 的大小. 我们有 $\operatorname{non}^{* *}(\operatorname{Fin} \times \operatorname{Fin})=\mathfrak{d}$ 并 且 $\operatorname{non}^{* *}(\mathcal{E D})=\operatorname{cov}(\mathcal{M})$. 同时我们将得到对任意的不满足 Fin-BW 性质的理想 $\mathcal{I}$, 都有 $\operatorname{non}^{* *}(\mathcal{I}) \geqslant \mathfrak{d}$.

我们采用标准的集合论记号, 可见文献 $[8,9]$. 一个集合 $X$ 上的理想是指 $X$ 的一些子集形成的族, 满足对子集与有限并封闭, 我们将主要考虑自然数集 $\omega$ 上的理想, 以 Fin 表示所有自然数的有限子集 所形成的理想, 即 Fin $=[\omega]^{<\omega}$. 我们假定所考虑的理想都是真理想 $($ 即 $\neq \mathcal{P}(\omega)$ ), 并且包含了所有的有 限子集.

如果 $\mathcal{I}$ 是 $\omega$ 上的一个理想, 则记 $\mathcal{I}^{+}=\mathcal{P}(\omega) \backslash \mathcal{I}$ 及 $\mathcal{I}^{*}=\{\omega \backslash A: A \in \mathcal{I}\}$.

对偶的、有滤子的概念, 自然数的一个子集族称为是滤子, 如果其满足对有限交与扩集封闭, 同样 我们在这里只谈论真的滤子并且包含所有的余有限集. 如果一个滤子还满足 $\forall A, B \in \mathcal{P}(\omega)(A \cup B \in$ $\mathcal{F} \Rightarrow A \in \mathcal{F} \vee B \in \mathcal{F}$ ), 那么 $\mathcal{F}$ 就称为是一个超滤 ( $\omega$ 上的). 设 $\mathcal{U}$ 是一个超滤, 如果对自然数集的任一 个分划 $\left\langle A_{n}: n \in \omega\right\rangle$ 满足对每个 $n, A_{n} \notin \mathcal{U}$, 都一定存在一个 $B \in \mathcal{U}$ 使得对每个 $n,\left|B \cap A_{n}\right|<\omega(\leqslant 1)$, 那么我们称 $\mathcal{U}$ 是一个 P- 点 (Ramsey 超滤).

设 $\mathcal{I}, \mathcal{J}$ 是 $\omega$ 上的两个理想, $\mathcal{I}$ 与 $\mathcal{J}$ 的 Fubini 乘积是 $\omega \times \omega$ 上的一个理想, 定义为 $A \in \mathcal{I} \times \mathcal{J}$ 当 且仅当 $\{n \in \omega:\{m:\langle n, m\rangle\} \notin \mathcal{J}\} \in \mathcal{I}$.

如果 $A, B \in[\omega]^{\omega}, A \subseteq^{*} B$ 表示 $|A \backslash B|<\omega$. 设 $\mathcal{I}$ 是 $\omega$ 上的一个理想, 下面的这些基数不变量定 义见文献 [10].

定义 1.2 设 $\mathcal{I}$ 是 $\omega$ 上的理想, 那么

$$
\begin{aligned}
& \operatorname{add}^{*}(\mathcal{I})=\min \left\{|\mathcal{A}|: \mathcal{A} \subseteq \mathcal{I} \wedge(\neg \exists B \in \mathcal{I})(\forall A \in \mathcal{A})\left(A \subseteq^{*} B\right)\right\}, \\
& \operatorname{non}^{*}(\mathcal{I})=\min \left\{|\mathcal{A}|: \mathcal{A} \subseteq[\omega]^{\omega} \wedge(\forall B \in \mathcal{I})(\exists A \in \mathcal{A})(|A \cap B|<\omega)\right\}, \\
& \operatorname{cov}^{*}(\mathcal{I})=\min \left\{|\mathcal{A}|: \mathcal{A} \subseteq \mathcal{I} \wedge\left(\forall B \in[\omega]^{\omega}\right)(\exists A \in \mathcal{A})(|A \cap B|=\omega)\right\}, \\
& \operatorname{cof}^{*}(\mathcal{I})=\min \left\{|\mathcal{A}|: \mathcal{A} \subseteq \mathcal{I} \wedge(\forall B \in \mathcal{I})(\exists A \in \mathcal{A})\left(B \subseteq^{*} A\right)\right\} .
\end{aligned}
$$

注意到如果 $\mathcal{I}$ 是一个极大理想, 那么 $\operatorname{add}^{*}(\mathcal{I}), \operatorname{non}^{*}(\mathcal{I}), \operatorname{cov}^{*}(\mathcal{I}), \operatorname{cof}^{*}(\mathcal{I})$ 已经在文献 $[11]$ 中出现 过了.

支配数 $\mathfrak{d}$ 和第一纲集的覆盖数 $\operatorname{cov}(\mathcal{M})$ 定义为

$$
\begin{aligned}
& \mathfrak{d}=\min \left\{|A|: A \subseteq \omega^{\omega} \wedge\left(\forall f \in \omega^{\omega}\right)(\exists g \in A)\left(\forall^{\infty} n\right)(g(n)>f(n))\right\} \\
& \operatorname{cov}(\mathcal{M})=\min \left\{|\mathcal{A}|: \mathcal{A} \subseteq \mathcal{M}\left(2^{\omega}\right) \wedge \cup \mathcal{A}=2^{\omega}\right\}
\end{aligned}
$$

这里 $\mathcal{M}\left(2^{\omega}\right)$ 指 $2^{\omega}$ 上的所有第一纲集所形成的集族.

一个集族 $\mathcal{A} \subseteq[\omega]^{\omega}$ 具有强有限交性质 (SFIP) 是指对所有的 $\mathcal{B} \in[\mathcal{A}]^{<\omega}, \cap \mathcal{B}$ 是一个无限集.

\section{2 与 $\mathcal{I}$ - 超滤有关的一个基数不变量}

定义 2.1 设 $\mathcal{I}$ 是 $\omega$ 上的一个理想, 我们定义下面的与 $\mathcal{I}$ 有关的基数不变量为

$$
\operatorname{non}^{* *}(\mathcal{I})=\min \left\{|\mathcal{A}|: \mathcal{A} \subseteq \mathcal{I}^{+} \wedge \mathcal{A} \text { 有强有限交性质, 并且 } \forall B \in \mathcal{I}, \exists A \in \mathcal{A},|A \cap B|<\omega\right\} .
$$


不难验证, $\operatorname{non}^{*}(\mathcal{I}) \leqslant \operatorname{non}^{* *}(\mathcal{I}) \leqslant \operatorname{cof}^{*}(\mathcal{I})$, 并且如果 $\mathcal{I} \subseteq \mathcal{J}$, 那么 $\operatorname{non}^{* *}(\mathcal{I}) \leqslant \operatorname{non}^{* *}(\mathcal{J})$. 当 $\mathcal{I}$ 是一 个极大理想时, $\operatorname{non}^{* *}(\mathcal{I})=\operatorname{cof}^{*}(\mathcal{I})=\chi(\mathcal{I})$.

定理 2.2 设 $\mathcal{I}$ 是 $\omega$ 上的一个理想, 那么我们有

(1) 如果 $\operatorname{non}^{* *}(\mathcal{I})=\mathfrak{c}$, 那么任何一个由小于 $\mathfrak{c}$ 个集合生成的滤子都包含在某个 $\mathcal{I}$ - 超滤中.

(2) 存在一个滤子刚好可以由 $\operatorname{non}^{* *}(\mathcal{I})$ 个集合生成, 但不包含在任何一个 $\mathcal{I}$ - 超滤中.

(3) 任何一个由小于 non** $(\mathcal{I})$ 个集合生成的超滤一定是 $\mathcal{I}$ - 超滤.

证明 (1) 设 $\operatorname{non}^{* *}(\mathcal{I})=\mathfrak{c}$, 并且 $\left\langle f_{\alpha}: \alpha<\mathfrak{c}\right\rangle$ 列出了所有的 $\omega_{\omega} \omega$ 中的函数. 设 $\kappa<\operatorname{non}^{* *}(\mathcal{I})$ 并且 $|\mathcal{F}|=\kappa$ 是一个滤子基, 不妨假设 $\mathcal{F}$ 对有限交封闭. 那么来构造一个递增的集族序列如下, $\left\langle\mathcal{F}_{\alpha}: \alpha<\mathfrak{c}\right\rangle$ : $\mathcal{F}_{0}=\mathcal{F}$, 并且如果 $\alpha$ 是一个极限序数, 则 $\mathcal{F}_{\alpha}=\bigcup_{\beta<\alpha} \mathcal{F}_{\beta}$. 对于后继序数 $\alpha+1$ : 如果存在一个 $F \in \mathcal{F}_{\alpha}$ 使得 $f_{\alpha}[F] \in \mathcal{I}$, 则令 $\mathcal{F}_{\alpha+1}=\mathcal{F}_{\alpha}$. 反之, 则有 $\forall F \in \mathcal{F}_{\alpha}\left(f_{\alpha}[F] \in \mathcal{I}^{+}\right)$. 那么 $\left\{f_{\alpha}[F]: F \in \mathcal{F}_{\alpha}\right\} \subseteq \mathcal{I}^{+}$ 有强有限交性质. 根据归纳假设及 $\operatorname{non}^{* *}(\mathcal{I})$ 的定义, $\left|\mathcal{F}_{\alpha}\right|<\operatorname{non}^{* *}(\mathcal{I})$, 存在一个 $B \in \mathcal{I}$ 使得对任意的 $F \in \mathcal{F}_{\alpha}$ 都有 $\left|B \cap f_{\alpha}[F]\right|=\omega$. 则我们取 $\mathcal{F}_{\alpha+1}=\mathcal{F}_{\alpha} \cup\left\{f_{\alpha}^{-1}[B]\right\}$. 那么任何一个包含 $\bigcup_{\alpha<\mathfrak{c}} \mathcal{F}_{\alpha}$ 的超滤 一定是一个 $\mathcal{I}$ - 超滤.

(2) 设 $\mathcal{A} \subseteq \mathcal{I}^{+}$具有强有限交性质, 并且满足 non** $(\mathcal{I})$ 的定义中的性质并且 $|\mathcal{A}|=\operatorname{non}^{* *}(\mathcal{I})$. 那么 任何一个包含 $\mathcal{A}$ 的超滤肯定不是 $\mathcal{I}$ - 超滤. 事实上对任何一个 $B \in \mathcal{I}$, 都有一个 $A \in \mathcal{A}$ 使得 $|B \cap A|<\omega$, 则 $B$ 一定不在这个超滤中, 于是恒等函数就见证了这个超滤肯定不是 $\mathcal{I}$ - 超滤.

(3) 完全类似于 (1) 的证明, 对任意的 $f \in{ }^{\omega} \omega$ 都有一个 $B \in \mathcal{I}$ 使得对任意的 $U \in \mathcal{U}, B \cap f(U)$ 是 无限的, 那么我们有 $f^{-1}[B] \in \mathcal{U}$ 并且 $f\left[f^{-1}[B]\right] \subseteq B \in \mathcal{I}$.

Flašková ${ }^{[4]}$ 指出了下面的关于 P- 点和 Ramsey 超滤的刻画.

定理 2.3 对任意的超滤 $\mathcal{U}$,

(1) $\mathcal{U}$ 是一个 P- 点当且仅当 $\mathcal{U}$ 是一个 Fin $\times$ Fin- 超滤.

$(2) \mathcal{U}$ 是一个 Ramsey 超滤当且仅当 $\mathcal{U}$ 是一个 $\mathcal{E} D$ - 超滤.

这里理想 $\mathcal{E D}$ 是 $\omega \times \omega$ 上的由坚直截口及函数的图形所生的一个理想, 即

$$
\mathcal{E D}=\{A \subseteq \omega \times \omega: \exists m, n \in \omega, \forall k>n,|\{l:\langle k, l\rangle \in A\}| \leqslant m\} .
$$

显然我们有 $\mathcal{E D} \subseteq$ Fin $\times$ Fin.

在下一节中我们将指出 $\operatorname{non}^{* *}(\operatorname{Fin} \times \operatorname{Fin})=\mathfrak{d}$ 并且 $\operatorname{non}^{* *}(\mathcal{E D})=\operatorname{cov}(\mathcal{M})$. 于是我们的结果可以看 成是对关于 P- 点及 Ramsey 超滤的一个推广.

\section{3 一些具体的理想的 $\operatorname{non}^{* *}(\mathcal{I})$}

我们将利用下面有用的引理, 证明可见文献 [7].

引理 3.1 设 $\left\langle C_{n}: n \in \omega\right\rangle$ 是一列递减的 $\omega$ 的子集序列, 并且 $\mathcal{A}$ 是一族由小于 $\mathfrak{d}$ 个自然数的无 穷子集所形成的集族, 满足对任意的 $A \in \mathcal{A}$ 都与每个 $C_{n}$ 交于无穷. 则存在一个 $B$ 使得对每个 $n$ 都 有 $B \subseteq^{*} C_{n}$ 成立, 并且 $B$ 与 $\mathcal{A}$ 中的每个元都交于无限.

$\omega$ 上的一个理想 $\mathcal{I}$ 满足 Fin-BW 性质是指对任意的有界的实数序列 $\left\langle x_{n}: n \in \omega\right\rangle$, 都存在一个 $A \notin \mathcal{I}$ 使得 $\left\langle x_{n}: n \in A\right\rangle$ 是收敛的.

关于 Fin-BW 性质的以下刻画可见文献 [12]. 
引理 3.2 设 $r \in \omega$. 一个理想满足 Fin-BW 性质当且仅当对任意的一个满足下列性质的集列 $\left\{A_{s}: s \in r^{<\omega}\right\}$ : (i) $A_{\emptyset}=\omega$; (ii) $A_{s}=\bigcup_{i<r} A_{s \frown i}$; (iii) $A_{s \frown i} \cap A_{s \frown j}=\emptyset$ 都存在一个 $x \in r^{\omega}$ 并且 $B \in \mathcal{I}^{+}$ 使得 $B \subseteq^{*} A_{x \uparrow n}$.

现在我们对不满足 Fin-BW 性质的理想 $\mathcal{I}$ 给出 $\operatorname{non}^{* *}(\mathcal{I})$ 的一个下界.

引理 3.3 如果 $\mathcal{I}$ 不满足 Fin-BW 性质, 则 $\operatorname{non}^{* *}(\mathcal{I}) \geqslant \mathfrak{d}$.

证明 设 $\left\{A_{s}: s \in r^{<\omega}\right\}$ 具有性质: (i) $A_{\emptyset}=\omega$; (ii) $A_{s}=\bigcup_{i<r} A_{s \frown i}$; (iii) $A_{s \frown i} \cap A_{s \frown j}=\emptyset$ 并且 $\forall x \in r^{\omega}, \forall n \in \omega, B \subseteq^{*} A_{x \mid n}$, 则 $B \in \mathcal{I}$.

设 $\mathcal{A} \subseteq \mathcal{I}^{+}$为一具有强有限交性质的集族, 并且基数小于 $\mathfrak{d}$. 随便取一个包含了 $\mathcal{A}$ 的超滤 $\mathcal{V}$, 则 易知存在一个 $x \in r^{\omega}$ 使得 $\forall n, A_{x \uparrow n} \in \mathcal{V}$. 由于 $\left\{A_{x \uparrow n}: n \in \omega\right\}$ 是一个递减的集列并且与 $\mathcal{A}$ 中的每个 集合交于无限. 由引理 3.1, 我们可以找到一个 $B$ 使得 $\forall n B \subseteq^{*} A_{x \uparrow n}$ 并且 $B$ 与 $\mathcal{A}$ 的每个集合交于无 限. 根据集族 $\left\{A_{s}\right\}$ 的性质, 我们有 $B \in \mathcal{I}$. 则 $B$ 见证了 $\operatorname{non}^{* *}(\mathcal{I}) \geqslant \mathfrak{d}$.

密度理想 $\mathcal{Z}_{0}$ 是由自然数的所有具有上密度为 0 的子集所形成的理想, 即

$$
\mathcal{Z}_{0}=\left\{A \subseteq \omega: \lim _{n \rightarrow \infty} \sup \frac{|A \cap n|}{n}=0\right\}
$$

我们记 $\operatorname{nwd}(\mathbb{Q})$ 为所有的有理数 $\mathbb{Q}$ 的无处稠密集所形成的理想, 这是有理数集 $\mathbb{Q}$ 上的一个理想.

引理 3.4 理想 $\mathcal{Z}_{0}$, Fin $\times$ Fin 和 $\operatorname{nwd}(\mathbb{Q})$ 都不具有 Fin-BW 性质.

证明可直接利用引理 3.3 对于 $\mathcal{Z}_{0}$ 和 $F$ in $\times$ Fin, 我们可分别直接取

$$
A_{s}=\left\{n \in \omega: n \equiv 2^{\sum_{i<|s|} s(i)}\left(\bmod 2^{|s|}\right)\right\}, \quad\left\{A_{s} \times \omega: s \in 2^{<\omega}\right\} .
$$

对于 n.w.d 则利用 $\mathbb{Q}$ 是零维的并且任何一个基本开集可分成两个基本开集的不交并.

推论 $3.5 \operatorname{non}^{* *}\left(\mathcal{Z}_{0}\right) \geqslant \mathfrak{d}, \operatorname{non}^{* *}(\operatorname{Fin} \times$ Fin $) \geqslant \mathfrak{d}, \operatorname{non}^{* *}(\operatorname{nwd}(\mathbb{Q})) \geqslant \mathfrak{d}$.

Hernández-Hernádez 和 Hrušák ${ }^{[10]}$ 提出如下问题: 是否 $\operatorname{cov}^{*}\left(\mathcal{Z}_{0}\right) \leqslant \mathfrak{b}$ 成立? 对 $\mathcal{Z}_{0}$ 结果可以看成 是这个问题的对偶命题 $\operatorname{non}^{*}\left(\mathcal{Z}_{0}\right) \geqslant \mathfrak{d}$ 的一个弱的形式.

对于理想 Fin $\times$ Fin, 我们指出其不等式可以反过来, 有下面的定理.

定理 3.6 $\operatorname{non}^{* *}(\operatorname{Fin} \times$ Fin $)=\operatorname{cof}^{*}(\operatorname{Fin} \times$ Fin $)=\mathfrak{d}$.

证明 我们只需证明 $\operatorname{cof}^{*}(\operatorname{Fin} \times$ Fin $) \leqslant \mathfrak{d}$. 设 $\left\{f_{\alpha} \in{ }^{\omega} \omega: \alpha<\mathfrak{d}\right\}$ 是一个支配族. 定义 $A_{\alpha}=$ $\left\{(x, y) \in \omega \times \omega: y<f_{\alpha}(x)\right\}$ 及 $B_{n}=n \times \omega$, 则显然有 $\mathcal{A}=\left\{A_{\alpha}: \alpha<\mathfrak{d}\right\} \cup\left\{B_{n}: n<\omega\right\} \subseteq$ Fin $\times$ Fin. 对任意的 $B \in \omega \times \omega$, 存在一个 $m \in \omega$ 及 $g \in{ }^{\omega} \omega$ 使得 $B \subseteq(m \times \omega) \cup\{(x, y) \in \omega \times \omega: y<g(x)\}$. 那么 存在一个 $\alpha<\mathfrak{d}$ 使得 $\forall n, f_{\alpha}(n)>g(n)$. 那么 $B \subseteq A_{\alpha} \cup B_{m}$. 这说明 $\mathcal{A}$ 生成了理想 Fin $\times$ Fin.

定理 $3.7 \operatorname{non}^{* *}(\mathcal{E D})=\operatorname{cov}(\mathcal{M}), \operatorname{cof}^{*}(\mathcal{E D})=\mathfrak{c}$.

证明 证明分为以下四部分进行:

(1) $\operatorname{non}^{* *}(\mathcal{E D}) \geqslant \operatorname{cov}(\mathcal{M})$;

(2) $\operatorname{non}^{* *}(\mathcal{E D}) \leqslant \mathfrak{d}$;

(3) $\operatorname{non}^{* *}(\mathcal{E D}) \leqslant \operatorname{cov}(\mathcal{M})$

(4) $\operatorname{cof}^{*}(\mathcal{E D}) \geqslant \mathfrak{c}$.

(1) 取定 $\kappa<\operatorname{cov}(\mathcal{M})$ 并且设 $\left\{A_{\alpha}: \alpha<\kappa\right\} \subseteq \mathcal{E D}^{+}$具有强有限交性质. 令 $C_{n}=(\omega \backslash n) \times \omega$, 由引 理 3.1, 我们可找到一个 $B$ 是所有 $\left\{C_{n}: n \in \omega\right\}$ 的伪交并且与每个 $A_{\alpha}$ 相交于无限. 取 $B_{n}=\{m \in \omega$ : $(n, m) \in B\}$, 则每个 $B_{n}$ 都是有限的. 将 $B$ 视为 $\prod_{n \in \omega} B_{n}$ 的乘积空间, 其与 Cantor 空间 $2^{\omega}$ 是同胚 
的. 则我们令

$$
X_{\alpha}=\left\{x \in \prod_{n \in \omega} B_{n}: \exists^{\infty} n \in \omega(n, x(n)) \in B \cap A_{\alpha}\right\} .
$$

不难验证知对每个 $\alpha<\operatorname{cov}(\mathcal{M}), X_{\alpha}$ 是稠密的 $G_{\delta}$ 集, 于是存在一个 $x \in \prod_{n \in \omega} B_{n}$ 属于所有的 $X_{\alpha}$. 则 $\{(n, x(n): n \in \omega)\} \in \mathcal{E D}$, 并且与每个 $A_{\alpha}$ 相交于无限集.

(2) 这是显然的, 因为我们有 $\operatorname{non}^{* *}(\mathcal{E D}) \leqslant \operatorname{non}^{* *}($ Fin $\times$ Fin $)=\mathfrak{d}$.

(3) 利用文献 [7] 中的方法, 取定 $\kappa<\operatorname{non}^{* *}(\mathcal{E D})$ 并且令 $\left.\left\{\left(x_{\alpha}, \Pi_{\alpha}\right): \alpha<\kappa\right)\right\}$ 为一族 chopped 实数, 其中 $\Pi_{\alpha}=\left\langle I_{\alpha n}: n \in \omega\right\rangle$.

所谓 chopped 实数是指一个有序对 $(x, \Pi)$, 其中 $x \in{ }^{\omega} 2, \Pi$ 为一区间分划. $\left(x,\left\langle I_{n}\right\rangle\right)$ 吞食了 $\left(y,\left\langle J_{n}\right\rangle\right)$ 是指 $\forall^{\infty} n \in \omega, \exists m \in \omega$ 使得 $I_{n} \supseteq J_{m}$ 且 $x \uparrow J_{m}=y \uparrow J_{m}$.

通过取有限交, 可以不妨假设其中的任意有限个 chopped 实数都可以被这族 chopped 实数中的另 外一个吞食. 可将 $\omega \times \omega$ 当成是 $\omega \times 2^{<\omega}$, 事实上它们有相同的基数.

由于 $\kappa<\mathfrak{d}$, 存在一个区间分划 $\left\{J_{n}: n \in \omega\right\}$ 使得

$$
\forall \alpha<\kappa, \quad \exists^{\infty} m \in \omega, \quad \exists n \in \omega, \quad I_{\alpha m} \subseteq J_{n} \cup J_{n+1} .
$$

定义

$$
A_{\alpha}=\left\{(n, s): s \in 2^{J_{n} \cup J_{n+1}} \wedge \exists m \in \omega\left(I_{\alpha m} \subseteq J_{n} \cup J_{n+1} \wedge s \uparrow I_{\alpha m}=x_{\alpha} \uparrow I_{\alpha m}\right)\right\} .
$$

由假设, 任意有限个 $\left(x_{\alpha}, \Pi_{\alpha}\right)$ 都可被其中另外一个吞食, 不难验证 $\left\{A_{\alpha}\right\}$ 有强有限交性质.

根据 $\operatorname{non}^{* *}(\mathcal{I})$ 的定义, 有 $B \in \mathcal{E D}$ 与每个 $A_{\alpha}$ 交于无限. 而只有有限个 $2^{J_{n} \cup J_{n+1}}$, 于是存在一个 $m \in \omega$ 及 $y_{0}, y_{1}, \ldots, y_{m-1}: \omega \rightarrow 2^{<\omega}$ 满足 $B=\bigcup_{i<m}\left\{\left(n, y_{i}(n)\right): n \in \omega\right\}$. 则其中必定有一个与每一 个 $A_{\alpha}$ 交于无限, 可不妨假设是 $y_{0}$. 则对所有的 $n, \operatorname{dom}\left(y_{0}(n)\right)=J_{n} \cup J_{n+1} . X_{0}=\left\{\left(2 n, y_{0}(2 n)\right): n \in \omega\right\}$ 和 $X_{1}=\left\{\left(2 n+1, y_{0}(2 n+1)\right): n \in \omega\right\}$ 中的一个与每个 $A_{\alpha}$ 相交于无穷. 通过对 $X_{i}$ 取并, 我们就找到 了 $z \in 2^{\omega}$ 使得 $\forall \alpha<\kappa, \exists^{\infty} n\left(z\left\lceil I_{\alpha n}=x_{\alpha} \uparrow I_{\alpha n}\right)\right.$ 成立.

(4) 由于可构造出一个集合 $\left\{f_{\alpha}: \alpha<\mathfrak{c}\right\} \subseteq \omega^{\omega}$ 满足对任意不同的 $\alpha, \beta$ 都有 $f_{\alpha}$ 和 $f_{\beta}$ 是最终不同 的. 则对每个 $\alpha<\mathfrak{c}$, 可令

$$
\mathcal{A}=\left\{A_{\alpha}: \alpha<\mathfrak{c}\right\}, \quad A_{\alpha}=\{(n, f(n)): n \in \omega\}
$$

由于 $\mathcal{E D}$ 中的每个集合都最多只能包含有限个 $\mathcal{A}$ 中的集合, 那么可知 $\mathcal{E D}$ 的任意一组基一定有基数 c. 证毕.

注 在本文投稿后作者获悉, Brendle 和 Flašková 也同时独立的引入并研究了本文中所考虑的基 数不变量. 本文曾于 2012 年 1 月在日本神户大学举办的 “Forcing in Set Theory Seminar” 国际会议 上报告.

\section{参考文献}

1 Baumgartner J. Ultrafilters on $\omega$. J Symbolic Logic, 1995, 2: 624-639

2 Brendle J. Between P-points and nowheredense ultrafilters. Israel J Math, 1999, 113: 205-230

3 Flašková J. Ultrafilters and small sets, Ph.D Thesis. Prague: Charles University, 2006

4 Flašková J. Description of some ultrafilters via $\mathcal{I}$-ultrafilter. Preprint

5 Ketonen J. On the existence of P-points in the Stone-C̆ech compactification of integers. Fund Math, 1976, 92: 91-94 
6 Canjar R M. On the generic existence of special ultrafilters. Proc Amer Math Soc, 1990, 110: 233-241

7 Blass A. Combinatorial cardinal characteristics of the continuum. In: Foreman M, Kanamori A, eds. Handbook of Set Theory. New York: Springer, 2010: 395-489

8 Kunen K. Set Theory: An Introduction to Independence Proofs. Amsterdam-New York-Oxford: North Holland, 1980

9 Jech T. Set Theory: Perspectives in Mathematical Logic, 2nd ed. Berlin: Springer-Verlag, 1997

10 Hernández-Hernádez F, Hrušák M. Cardinal invariants of analytic P-ideals. Canad J Math, 2007, 59: 575-595

11 Brendle J, Shelah S. Ultrafilters on $\omega$-their ideals and their cardinal characteristics. Trans Amer Math Soc, 1999, 351: 2643-2674

12 Filipów R, Mrożek N, Reclaw I, et al. Ideal version of bounded sequence. J Symbolic Logic, 2007, 72: 501-512

\title{
Cardinal invariants related to the $\mathcal{I}$-ultrafilters
}

\author{
HONG JianYong \& ZHANG ShuGuo
}

Abstract We introduce a new cardinal invariants $\operatorname{non}^{* *}(\mathcal{I})$ for an ideal on $\omega$ which is showed to be closely related to the $\mathcal{I}$-ultrafilters. We prove the following results:

(1) If non $^{* *}(\mathcal{I})=\mathfrak{c}$ then each filter generated by fewer than $\mathfrak{c}$ sets is included in some $\mathcal{I}$-ultrafilter.

(2) There is a filter generated by non** $(\mathcal{I})$ sets that is not included in any $\mathcal{I}$-ultrafilter.

(3) Each ultrafilter generated by fewer than non** $(\mathcal{I})$ sets is an $\mathcal{I}$-ultrafilter.

These can be considered as a generalization of the classical results for P-point and Ramsey ultrafilter. We then estimate $\operatorname{non}^{* *}(\mathcal{I})$ for some ideals. We show that non ${ }^{* *}($ Fin $\times$ Fin $)=\mathfrak{d}, \operatorname{non}^{* *}(\mathcal{E D})=\operatorname{cov}(\mathcal{M})$ and non ${ }^{* *}(\mathcal{I}) \geqslant \mathfrak{d}$ for the ideals do not have the Fin-BW property.

Keywords ideals, $\mathcal{I}$-ultrafilter, cardinal invariant

MSC(2010) 03E17, 03E05

doi: $10.1360 / 012011-656$ 